\title{
Noise driven compressed sensing method for space time signal processing
}

\author{
Liu Jing ${ }^{\text {a) }}$ \\ School of Electronics and Information Engineering, Xi'an JiaoTong University, Xi'an, \\ China 710049 \\ a)elelj20080730@gmail.com
}

\begin{abstract}
In contrary to the existing work related with compressed sensing based STAP technique, which adopts the original sensing matrix, the proposed noise driven compressed sensing method is to construct a new sensing matrix with weak coherence through incorporating the measurement noise. The proposed method tries to build an equivalent system of the classical model in compressed sensing, resulting in an equivalent sensing matrix. Inspired by the idea that low coherence guarantees the reconstruction of the sparse vector with large probability, the equivalent sensing matrix is updated iteratively in a Markov chain Monte Carlo (MCMC) based framework to reduce the large coherence between a set of specific columns in the original sensing matrix. At the same time, the proposed method tries to preserve most of the information of the original sensing matrix via adjusting a noise related matrix. The simulation results show that the proposed method obtains much less average reconstruction error compared with the existing compressed sensing based STAP methods, and it is also very efficient when coping with measurement noise with low SNR.
\end{abstract}

Keywords: noise driven compressed sensing, STAP, airborne radar, Markov chain Monte Carlo (MCMC)

Classification: Microwave and millimeter wave devices, circuits, and systems

\section{References}

[1] J. Guerci, Space-time adaptive processing for radar, Artech House, 2003.

[2] R. Klemm, Applications of space-time adaptive processing, Inspec/IEE, 2004.

[3] W. Melvin, "A STAP overview," IEEE Aerosp. Electron. Syst. Mag., vol. 19, no. 1, pp. 19-35, 2004.

[4] H. Zhang, G. Li, and H. Meng, "A class of novel STAP algorithms using sparse recovery technique," Arxiv preprint arXiv:0904.1313., 2009.

[5] I. W. Selesnick, S. U. Pillai, K. Y. Li, and B. Himed, "Angle-Doppler processing using sparse regularization," Proc. IEEE Int. Conf. Acoustics, Speech and Signal Processing, 2010.

[6] J. Parker and L. Potter, "A Bayesian perspective on sparse regularization for STAP post-processing," IEEE Radar Conference, pp. 1471-1475, 
2010.

[7] K. Sun, H. Meng, and Y. Wang, "Direct data domain STAP using sparse representation of clutter spectrum," Signal Processing, vol. 91, no. 9, pp. 2222-2236, 2011.

[8] J. A. Tropp, "Greed is good: Algorithmic results for sparse approximation," IEEE Trans. Inf. Theory, vol. 50, no. 10, pp. 2231-2242, 2004.

[9] M. F. Duarte and Y. C. Eldar, "Structured compressed sensing: From theory to applications," IEEE Trans. Signal Process., vol. 59, no. 9, pp. 4053-4085, 2011.

[10] I. Stojanovic, W. Karl, and M. Cetin, "Compressed sensing of monostatic and multi-static SAR," SPIE Defense and Security Symposium, Algorithms for Synthetic Aperture Radar Imagery XVI.

[11] J. H. G. Ender, "On compressive sensing applied to radar," Signal Processing, vol. 90, no. 5, pp. 1402-1414, 2010.

[12] W. R. Gilks, S. Richardson, and D. J. Spiegelhalter, Markov Chain Monte Carlo in Practice, Chapman and Hall, London, 1996.

[13] B. Himed, Y. Zhang, and A. Hajjari, "STAP with angle-Doppler compensation for bistatic airborne radars," Proc. IEEE National Radar Conference, LongBeach, California, pp. 22-25, 2002.

[14] R. Klemm, Principles of Space-Time Adaptive Processing, IEE Press, 1999.

\section{Introduction}

STAP is a signal processing technique that was originally developed for detecting slowly moving targets using airborne radars. It represents the simultaneous adaptive application of both Doppler filtering and spatial beamforming $[1,2]$, and allows the suppression of clutter that neither technique could individually address. While much of the early work in STAP focuses on the simplest case of side-looking uniform linear arrays (ULAs) operating monostatically, STAP techniques have also been applied to bistatic systems, conformal arrays, space-based systems, and other applications [3]. However, the traditional STAP algorithm uses a lot of training cells to estimate the space-time covariance matrix, which occupies large computer memory and is time-consuming.

In recent years, a number of compressed sensing based methods are proposed to detect unknown moving targets in strong clutter situation directly on the space-time data, which reduces the measurement data efficiently $[4,5,6,7]$. In [4], the entire radar scene, DOA-Doppler plane, is reconstructed using a compressed sensing based approach, and an attempt is then made to identify and zero out the clutter component. In [5] the problem of clutter is addressed by applying a mask to the signal in the DOA-Doppler plane before penalizing, and the proposed method is shown to outperform the sample matrix inversion (SMI) method [1]. However, it is based on the assumption of known clutter ridge location. The work in [6] is a combination of the traditional STAP algorithm and compressed sensing. The STAP is used to suppress the clutter, and the compressed sensing is then utilized to 
reconstruct the entire radar scene which only contains the targets. In [7], by exploiting the intrinsic sparsity of the spectral distribution, a new direct data domain approach using sparse representation (D3SR) is proposed, which seeks to estimate the high-resolution space-time spectrum with only the test cell. However, the method assumes that the area where the targets locate is known in prior.

The classical model of compressed sensing, $y=\Phi x+e$, is adopted in the above work. The measurement vector $y$ represents the received echo signal snapshot from fixed range cell, and $x$ is the collection of the strength of the original transmitted signals (including targets, clutter or both) from the whole DOA-Doppler plane. $e$ denotes the measurement noise. The sensing matrix $\Phi$ is comprised of Spatial-Doppler steering vectors in column, which is deterministic in nature. All the above work assumes that the sparse vector $x$ could be reconstructed based on the sensing matrix $\Phi$ perfectly. However, the coherence of the sensing matrix is not low and does not guarantee a good reconstruction of the sparse vector with large probability according to $[8,9]$. The simulation results in Section 5 in this paper show that large reconstruction error exists when relying on the original sensing matrix $\Phi$.

In this paper, a novel method named as noise driven compressed sensing is proposed to decrease the reconstruction error via reducing the coherence of the original sensing matrix $\Phi$. The proposed method tries to build an equivalent system of the classical model in compressed sensing via incorporating the measurement noise $e$, which results in an equivalent sensing matrix $\Phi_{E}$. Inspired by the idea that low coherence guarantees the reconstruction of the sparse vector with large probability $[8,9]$, the equivalent sensing matrix $\Phi_{E}$ is updated iteratively in an MCMC based framework to reduce the large coherence between a set of specific columns in the original sensing matrix $\Phi$. At the same time, the proposed method tries to preserve most of the information of $\Phi$ via adjusting a noise related matrix.

In this paper, we consider the application of detecting unknown moving targets in strong clutter situation using airborne radar system. Since the airborne radar scenario has a high CSR (clutter-signal-ratio, $>20 \mathrm{~dB}$ ), the prominent elements of the spectral distribution focus along the clutter ridge in the DOA-Doppler plane. Therefore, it is reasonable to assume that the received data of the test cell is sparse in the DOA-Doppler plane [7]. The proposed noise driven compressed sensing method is then used to reconstruct the sparse signal representing the radar scene. From the simulation results, it can be seen that both the prominent elements (the clutter) and the weak elements (targets) are recovered accurately in the reconstructed DOA-Doppler plane, and consequently the targets are distinguished from the clutter successfully. This is due to the reason that the proposed noise driven compressed sensing method can cope with the deterministic sensing matrix with high coherence efficiently.

The paper is organized as follows: Section 2 introduces the general spacetime model, which is represented in a compressed sensing framework. Our main contribution, the noise driven compressed sensing method, is introduced 
in Section 3, and the compressed sensing based multiple targets detection algorithm is introduced in Section 4. The simulation results are listed in Section 5, and the paper is summarized in Section 6.

\section{A general space-time model and its sparse representation}

In this paper, we consider an airborne radar system which transmits $K$ coherent pulse trains and samples the returns on ULAs consisting of $N$ elements. For each pulse, it collects $Q$ temporal samples from each element receiver, where each time sample corresponds to a range cell. The collection of samples for the $q^{\text {th }}$ range cell is represented by an $N \times K$ data matrix $\mathbf{F}$ (snapshot) with elements $f(n, k)$ as,

$$
\mathbf{F}=\left[\begin{array}{ccc}
f(1,1) & f(1,2) & \cdots f(1, K) \\
f(2,1) & f(2,2) & \cdots f(2, K) \\
\cdots & \cdots & \cdots \\
f(N, 1) & f(N, 2) & \cdots f(N, K)
\end{array}\right]
$$

A test cell is assumed to be comprised of target and clutter components. First, assuming $D$ targets are observed in the far-field, the $i^{\text {th }}$ target is at a DOA angle of $\theta_{i}^{t}$ with Doppler frequency $f_{d_{i}}^{t}$. We can obtain an $N K \times 1$ complex vector $\mathbf{y}_{\mathbf{t}}$ as,

$$
\mathbf{y}_{\mathbf{t}}=\Sigma_{i=1}^{D} \beta\left(\theta_{i}^{t}, f_{d_{i}}^{t}\right)\left[\mathbf{s}_{\mathbf{S}}\left(\theta_{i}^{t}\right) \otimes \mathbf{s}_{\mathbf{T}}\left(f_{d_{i}}^{t}\right)\right],
$$

where $\beta\left(\theta_{i}^{t}, f_{d_{i}}^{t}\right)$ is the reflection coefficient of the $i^{\text {th }}$ target, ' $\otimes$ ' represents the Kronecker product of two vectors. The spatial steering vector $\mathbf{s}_{\mathbf{S}}\left(\theta_{i}^{t}\right)$ and the Doppler filtering steering vector $\mathbf{s}_{\mathbf{T}}\left(f_{d_{i}}^{t}\right)$ are represented by

$$
\mathbf{S}_{\mathbf{S}}\left(\theta_{i}^{t}\right)=\left[1, e^{j \frac{2 \pi d}{\lambda} \sin \theta_{i}^{t}}, \cdots, e^{j(N-1) \frac{2 \pi d}{\lambda} \sin \theta_{i}^{t}}\right]^{T}
$$

and,

$$
\mathbf{S}_{\mathbf{T}}\left(f_{d_{i}}^{t}\right)=\left[1, e^{j \frac{2 \pi f_{d_{i}}^{t}}{f_{r}}}, \cdots, e^{j(K-1) \frac{2 \pi f_{d_{i}}^{t}}{f_{r}}}\right]^{T},
$$

where $d$ is the distance between the elements of the arrays, $\lambda$ and $f_{r}$ denote wavelength and pulse repetition frequency, respectively.

Besides the target components, there also exists clutter component $\mathbf{y}_{\mathbf{c}}$, which can be considered as a collection of independent scatters as,

$$
\mathbf{y}_{\mathbf{c}}=\Sigma_{i=1}^{N_{c}} \beta\left(\theta_{i}^{c}, f_{d_{i}}^{c}\right)\left[\mathbf{s}_{\mathbf{S}}\left(\theta_{i}^{c}\right) \otimes \mathbf{s}_{\mathbf{T}}\left(f_{d_{i}}^{c}\right)\right],
$$

where $N_{c}$ is the number of clutter scatters. $\theta_{i}^{c}$ and $f_{d_{i}}^{c}$ are the DOA angle and Doppler frequency for the $i^{t h}$ clutter scatter respectively, and $\beta\left(\theta_{i}^{c}, f_{d_{i}}^{c}\right)$ is the reflection coefficient. $\mathbf{s}_{\mathbf{S}}\left(\theta_{i}^{c}\right)$ and $\mathbf{s}_{\mathbf{T}}\left(f_{d_{i}}^{c}\right)$ represent the spatial steering vector and the Doppler filtering steering vector respectively.

Using the above modeling, the $N K \times 1$ complex vector of the test cell can be modeled as

$$
\mathbf{y}_{\text {test }}=\mathbf{y}_{\mathbf{t}}+\mathbf{y}_{\mathbf{c}}+\mathbf{e}
$$

where $\mathbf{e}$ is an $N K \times 1$ complex Gaussian noise vector. 
In this paper, compressed sensing is used to estimate the spectral distributions of the targets and clutter scatters in the DOA-Doppler plane. To do so, the DOA-Doppler plane is divided into $V \times L$ square grids, where $V$ and $L$ denote the number of rows (for Doppler frequency) and columns (for DOA angle), respectively. Each grid is with the same size $\Delta \theta \times \Delta f_{d}$. Grid $(i, j)$ represents a DOA angle of $\theta_{i}\left(\theta_{0}+(i-1) \Delta \theta\right)$ and a Doppler frequency of $f_{d_{j}}\left(f_{d_{0}}+(i-1) \Delta f_{d}\right)$, where $\theta_{0}$ and $f_{d_{0}}$ represent the initial DOA angle and initial Doppler frequency. All the grids in the DOA-Doppler plane are mapped into a $2-\mathrm{D}$ vector $\mathbf{x}_{\text {test }}$ with the $j^{\text {th }}$ column put at the end of the $(j-1)^{\text {th }}$ column.

Since the airborne radar scene has a high CSR $(>20 \mathrm{~dB})$, the significant elements of the spectral distribution focus along the clutter ridge in the DOADoppler plane. Therefore, it is reasonable to assume that the received data of the test cell is sparse in the DOA-Doppler plane [7]. A small number of grids are occupied by the targets and clutter scatters in the DOA-Doppler plane, and $\mathbf{x}_{\text {test }}$ is a sparse vector.

Based on the above derivation, a system for the test cell is built in a compressed sensing framework as in (7),

$$
\mathbf{y}_{\text {test }}=\boldsymbol{\Phi} \mathbf{x}_{\text {test }}+\mathbf{e}
$$

$\boldsymbol{\Phi}$ is a sensing matrix with dimension $N K \times V L$, which is defined as $\boldsymbol{\Phi}=$ $\left[\begin{array}{llll}\boldsymbol{\varphi}_{1} & \boldsymbol{\varphi}_{2} & \cdots & \boldsymbol{\varphi}_{V L}\end{array}\right]$ in columns. The $((i-1) \cdot L+j)^{t h}$ column of $\boldsymbol{\Phi}$ is defined as follows,

$$
\boldsymbol{\varphi}_{(i-1) \cdot L+j}=\mathbf{s}_{\mathbf{S}}\left(\theta_{i}\right) \otimes \mathbf{S}_{\mathbf{T}}\left(f_{d_{j}}\right) .
$$

The sensing matrix $\boldsymbol{\Phi}$ is with high coherence since $V$ and $D$ are set large values to obtain a high resolution of the DOA-Doppler plane. Though in (7) the radar vectors and matrices are complex valued in contrary to the original compressed sensing environment, it is easy to transfer it to real variables according to $[10,11]$.

For simplicity, (7) is rewritten in a classical format in compressed sensing with subscripts removed.

$$
\mathbf{y}=\mathbf{\Phi} \mathbf{x}+\mathbf{e}
$$

\section{A heuristic noise driven compressed sensing method}

The existing compressed sensing based STAP methods assume that the sparse vector $x$ could be reconstructed based on the sensing matrix $\Phi$ perfectly. However, the coherence of the sensing matrix $\Phi$ is not low and does not guarantee a good reconstruction of the sparse vector with large probability. In contrast to the existing work, the proposed method tries to build an equivalent system of the classical model in compressed sensing (9) as follows,

$$
y=(\Phi+P) x,
$$

where

$$
P x=e .
$$


Since the dimension of $e$ is much less than that of $x,(11)$ is an underdetermined system and there exists a lot of matrix $P$ which satisfies (11). We simply choose

$$
P=e x^{\dagger}
$$

where $x^{\dagger}$ represents the pseudoinverse of $x$. We then let $\Phi_{E}=\Phi+P$, and (10) could be rewritten as

$$
y=\Phi_{E} x .
$$

$\Phi_{E}$ is defined as the equivalent sensing matrix. If $\Phi_{E}$ is with low coherence, it is possible to reconstruct $x$ from $\left(y, \Phi_{E}\right)$ with large probability using a number of $l_{1}$ minimization algorithms and greedy algorithms $[8,9]$. The coherence $\mu(\Psi)$ of a matrix $\Psi$ is defined as in [9],

$$
\mu(\Psi)=\max _{1 \leq i \neq j \leq N} \frac{\left|<\psi_{i}, \psi_{j}>\right|}{\left\|\psi_{i}\right\|_{2}|| \psi_{j} \|_{2}},
$$

where $\psi_{i}$ and $\psi_{j}$ represent the $i^{\text {th }}$ and the $j^{\text {th }}$ column of $\Psi$ respectively, and $N$ denotes the number of columns in $\Psi$.

However, the $K$-sparse vector $x$ is unknown and is to be reconstructed. Without loss of generality, it is assumed that the measurement noise $e$ distributes according to a Gaussian distribution $N\left(\mu, \sigma^{2}\right)$, where $\mu$ and $\sigma$ denote the mean and standard deviation respectively and they are assumed known in prior. It is unable to get the exact value of $P$ via (12) since both $x$ and $e$ are unknown. The following question arises: Given the deterministic sensing matrix $\Phi$, measurement vector $y$, how could we reconstruct $K$-sparse vector $x$ from the equivalent system (13)?

In this paper, an MCMC based algorithm is proposed to estimate the $K$-sparse vector $x$, the measurement noise $e$ and the matrix $P$ iteratively. Inspired by the idea that low coherence guarantees the reconstruction of the sparse vector with large probability, the equivalent sensing matrix $\Phi_{E}$ is updated iteratively in an MCMC based framework to reduce the large coherence between a set of specific columns. At each iteration of the proposed method, the coherence of the current equivalent sensing matrix $\Phi_{E}$ is evaluated and used as the acception/rejection criteria: the move resulting in a matrix with less coherence will be accepted with large probability, and vice verse. According to the theory of MCMC [12], this procedure would result in an equivalent sensing matrix with less coherence at each iteration. As a result, at the $(\tau+1)^{t h}$ iteration, the obtained estimate value of $x, x_{\tau+1}$, is more close to $x$ compared with $x_{\tau}$, which is obtained at the $\tau^{\text {th }}$ iteration. The estimate measurement noise $e_{\tau+1}$ would be more close to the true measurement noise $e$ as shown in (25). The proposed method also tries to preserve most of the information of the original sensing matrix $\Phi$ via adjusting the noise related matrix $P$. The detailed procedures of the proposed method are listed in the following:

\section{Algorithm 1: Noise driven compressed sensing:}


(1): Initializing, set

$$
\begin{aligned}
& \tau=0, \\
& x_{0}=\operatorname{Reconstruct}(\Phi, y) \text {, } \\
& \text { Sample } e_{0} \sim N\left(\mu, \sigma^{2}\right) \text {, } \\
& P_{0}=e_{0} x_{0}^{-1}, \\
& \Phi_{E, 0}=\Phi+P_{0}, \\
& E R R_{0}=y-\left(\Phi x_{0}+e_{0}\right) \text {. }
\end{aligned}
$$

(2): Obtain $\Phi_{E}^{\prime}$ via reducing the coherence of $\Phi_{E, \tau}$ (Algorithm 2),

$$
\Phi_{E}^{\prime}=\text { Reduce_Coherence }\left(\Phi_{E, \tau}\right) .
$$

(3): Sample $\rho \sim U(0,1)$, where $U(0,1)$ is a uniform distribution in the interval $(0,1)$.

(4): Calculate the acceptance ratio,

$$
a=\frac{\mu\left(\Phi_{E, \tau}\right)}{\mu\left(\Phi_{E}^{\prime}\right)} .
$$

(5): If $\rho \leq \min \{1, a\}$, then accept move:

$$
x_{\tau+1}=\text { Reconstruct }\left(\Phi_{E}^{\prime}, y\right),
$$

else reject move:

$$
x_{\tau+1}=\operatorname{Reconstruct}\left(\Phi_{E, \tau}, y\right) .
$$

The estimates of $e, P$ and $\Phi$ are updated as follows,

$$
\begin{aligned}
e_{\tau+1} & =y-\Phi x_{\tau+1}, \\
P_{\tau+1} & =e_{\tau+1} x_{\tau+1}^{-1}, \\
\Phi_{E, \tau+1} & =\Phi+P_{\tau+1} .
\end{aligned}
$$

(6): Calculate $E R R_{\tau+1}$ via (29) and do the following judgements.

If $E R R_{\tau+1}>T_{E R R}$ and $\tau<D$

$$
\tau=\tau+1, \text { Go to step (2); }
$$

Else

$$
\tau=\tau+1, \text { Goto step (7). }
$$

End

(7): Obtain the estimate value of $x$ as per (28).

$$
x_{\text {estimate }}=x_{\tau+1}
$$

In the above algorithm, 'Reconstruct' in (16) refers to any available reconstruction algorithm and the basis pursuit denoising (BPDN) method [9] is chosen as the reconstruction algorithm here. ' $E R R_{\tau+1}$ ' in Step (6) is defined as the error between the true measurement and the estimate measurement as follows,

$$
E R R_{\tau+1}=y-\left(\Phi x_{\tau+1}+e_{\tau+1}\right)
$$


If $E R R_{\tau+1}$ is less than the predefined threshold $T_{E R R}$, the iteration would be stopped with the resultant estimate $x_{\text {estimate }}(28)$. In Step (6), $D$ denotes the number of MCMC iterations.

The algorithm defined as Reduce_Coherence in Algorithm 1 is developed for updating the equivalent sensing matrix $\Phi_{E, \tau}$ iteratively while reducing the matrix's coherence. The idea is to find pairs of columns with large coherence (exceeding a predefined threshold $T_{c}$ ) in $\Phi_{E, \tau}$, and do Gram-Schmidt orthonormalization on each selected pair of columns, which results in a new matrix $\Phi_{E}^{\prime}$ with lower coherence (21). The value of $T_{c}$ should be set properly in order to obtain a new matrix $\Phi_{E}^{\prime}$ that is with lower coherence as well as keeping most of the information of $\Phi$. In the proposed algorithm, the value of $T_{c}$ is set large enough so that a small fraction of columns of $\Phi_{E, \tau}$ would be orthonormalizd while the other columns remains unchanged. The detailed procedure for the Reduce_Coherence algorithm is listed in Algorithm 2.

Algorithm 2: Reduce_coherence:

Input: an $M \times N$ matrix $\Psi$ and threshold $T_{c}$;

Output: an $M \times N$ matrix $\Psi^{\prime}$.

(1): Calculate the coherence between any two columns of the matrix $\Psi$,

$$
\mu\left(\psi_{i}, \psi_{j}\right)=\frac{\left|<\psi_{i}, \psi_{j}>\right|}{\left\|\psi_{i}\right\|_{2}|| \psi_{j} \|_{2}}, \quad i \neq j
$$

where $\psi_{i}$ and $\psi_{j}$ represent the $i^{\text {th }}$ and the $j^{\text {th }}$ column of $\Psi$ respectively, and $\langle a, b\rangle$ represents the inner product of two vectors $a$ and $b$.

(2): For all $\left\{\mu\left(\psi_{i}, \psi_{j}\right), i=1, \cdots M, j=1, \cdots, N\right.$, and $\left.i \neq j\right\}$, find pairs of columns $\left\{\psi_{\alpha}, \psi_{\beta}\right\}$ that satisfies

$$
\mu\left(\psi_{\alpha}, \psi_{\beta}\right) \geq T_{c}
$$

where $\psi_{\alpha}$ and $\psi_{\beta}$ represent the $\alpha^{\text {th }}$ and $\beta^{\text {th }}$ column of the matrix $\Psi$.

(3): For each pair of columns obtained from Step (2), $\left\{\psi_{\alpha}, \psi_{\beta}\right\}$, do the GramSchmidt orthonormalization and output the results directly to the matrix $\Psi^{\prime}$,

$$
\begin{aligned}
& \psi_{\alpha}^{\prime}=\psi_{\alpha}, \\
& \psi_{\beta}^{\prime}=\psi_{\beta}-\frac{<\psi_{\beta}, \psi_{\alpha}^{\prime}>}{<\psi_{\alpha}^{\prime}, \psi_{\alpha}^{\prime}>} \psi_{\alpha}^{\prime},
\end{aligned}
$$

where $\psi_{\alpha}^{\prime}$ and $\psi_{\beta}^{\prime}$ represent the $\alpha^{\text {th }}$ and $\beta^{\text {th }}$ column of the output matrix $\Psi^{\prime}$. And the remaining columns in $\Psi$ that do not satisfy (31) keep unchanged in the output matrix $\Psi^{\prime}$.

\section{Compressed sensing based multiple target detection algo- rithm}

In recent work related with compressed sensing based STAP $[4,5,6,7]$, the coherence of the sensing matrix is not low due to the high resolution of the DOA-Doppler plane, which does not guarantee a good reconstruction of the sparse vector with large probability. Consequently, the direct estimation of the target amplitude may be unreliable using sparse representation when 
locating a moving target from the surrounding strong clutter. The weak element (target) is always submerged in the prominent elements (the clutter). In [7], only the prominent elements are extracted from the sparse radar scene, and an additional adaptive filter is used to suppress the clutter to identify the target.

However, in this paper, we can obtain a reconstructed radar scene with high accuracy based on the test cell using the proposed noise driven compressed sensing algorithm. Both the prominent elements (the clutter) and the weak elements (multiple targets) can be identified accurately with the proposed method. Consequently, it is not difficult to distinguish the weak elements from the prominent elements in the reconstructed radar scene. In the following, a simple algorithm is proposed to detect multiple targets in the reconstructed radar scene.

\section{Algorithm 3: Compressed sensing based multiple target detection algorithm}

1. Use the proposed noise driven compressed sensing algorithm to obtain the estimate of the original sparse vector $\left(\mathbf{x}_{\text {test }}\right), \hat{\mathbf{x}}_{\text {test }}$, based on the original sensing matrix $\boldsymbol{\Phi}$, and the measurement vector $\mathbf{y}_{\text {test }}$ (the snapshot from the test cell). The entries corresponding to noise in the original sparse vector $\mathbf{x}_{\text {test }}$ are set as zero in $\hat{\mathbf{x}}_{\text {test }}$ according to the proposed algorithm. The nonzero elements of the reconstructed sparse vector $\hat{\mathbf{x}}_{\text {test }}$ contain the prominent elements (the clutter) and the weak elements (the targets).

2. Distinguish the weak elements from the prominent elements to detect multiple targets. For each element of the estimated sparse vector, $\hat{x}_{\text {test }}(i), i=1, \cdots, V D$, if $\left|\hat{x}_{\text {test }}(i)\right|>T_{\text {clutter }}$

$\hat{x}_{\text {test }}(i)$ corresponds to clutter.

else if $\left|\hat{x}_{\text {test }}(i)\right|>0$

$\hat{x}_{\text {test }}(i)$ corresponds to a target.

end

Here $T_{\text {clutter }}$ is a threshold set to distinguish the targets from the clutter. It is assumed that the CSR is sufficient large $(>20 d B)$. The clutter scatters are with much higher amplitudes than the targets. The entries of $\hat{\mathbf{x}}_{\text {test }}$ can be arranged in descend order according to their amplitudes as,

$$
\left|\hat{x}_{\text {test }}[1]\right|>\left|\hat{x}_{\text {test }}[2]\right|>\cdots>\left|\hat{x}_{\text {test }}[k]\right|>>\left|\hat{x}_{\text {test }}[k+1]\right|>\cdots>\left|\hat{x}_{\text {test }}[N K \times V L]\right|,
$$

where $\hat{x}_{\text {test }}[1]$ denotes the entry that with the largest amplitude, and the similar definitions for $\hat{x}_{t e s t}[2], \cdots, \hat{x}_{t e s t}[N K \times V L]$. The sudden change of the amplitude between $\hat{x}_{\text {test }}[k]$ and $\hat{x}_{\text {test }}[k+1]$ is caused by the large difference between the amplitudes of the clutter scatter and target element. And the value of $T_{\text {clutter }}$ is set proportional to the smallest amplitude of the clutter scatters, as $T_{\text {clutter }}=\kappa\left|\hat{x}_{\text {test }}[k]\right|$. The constant $\kappa$ is drawn from the range of $\left[10^{-2}, 10^{-1}\right]$ to ensure that the targets can be distinguish from the clutter 
scatters accurately. The proposed algorithm can identify multiple targets directly from the reconstructed radar scene (the DOA-Doppler plane), which reduces the computing complexity efficiently.

\section{Simulation results and analysis}

In this section, the proposed noise driven compressed sensing algorithm is compared with the D3SR method [7], in reconstructing sparse radar scenarios. Furthermore, the proposed algorithm is also compared with several classic STAP algorithms, e.g. the SMI method [1], the angle-Doppler compensation (ADC) method [13] and the D3SR method using the improvement factor loss $\left(I F_{\text {Loss }}\right)$, which is a common metric in evaluating the performance of the STAP methods.

An airborne, side-looking radar system consisting of half-wavelength spaced ULAs is considered in this section. The radar system is comprised of 16 arrays and the data is organized in CPIs of 16 pulses. The clutter is uniformly distributed between the directions of $-80^{\circ} \sim 70^{\circ}$ and is contained in both the training cells and the test cell. The DOA-Doppler plane is divided into $200 \times 180$ square grids, where $\mathrm{x}$-axis is for DOA angle and $\mathrm{y}$-axis for Doppler frequency. The initial DOA angle $\left(\theta_{0}\right)$, the DOA angle interval $(\Delta \theta)$, the initial Doppler frequency $\left(f_{d_{0}}\right)$ and the Doppler frequency interval $\left(\Delta f_{d}\right)$ equal $-90^{\circ}, 1^{\circ},-400 \mathrm{~Hz}$ and $4 \mathrm{~Hz}$, respectively.

First, the proposed algorithm is compared with the D3SR method in estimating targets with different positions under different noise levels. Ten targets distributes randomly in the DOA-Doppler plane. The reconstruction error is adopted to evaluate the reconstruction performance of the two methods, which is defined as

$$
\chi=\frac{\left\|x_{\text {estimate }}-x\right\|_{2}^{2}}{\|x\|_{2}^{2}},
$$

where $x$ and $x_{\text {estimate }}$ represent the true and estimated signal representing the sparse radar scene respectively. For a given signal-noise-ratio (SNR), we make 100 trails Monte Carlo simulations (indicated by $N_{M C}$ ). In each trail, the locations of ten targets are randomly distributed in the DOA-Doppler plane. Figs. 1 3 show the simulation results from one trial with SNR equaling 20 dB. Fig. 1 gives the actual sparse radar scene. Figs. $2 \sim 3$ provide the sparse

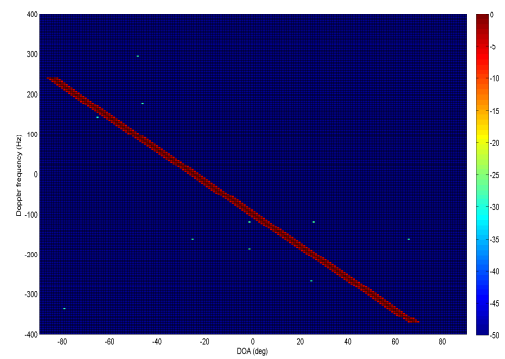

Fig. 1. True sparse radar scene: ten randomly distributed targets 


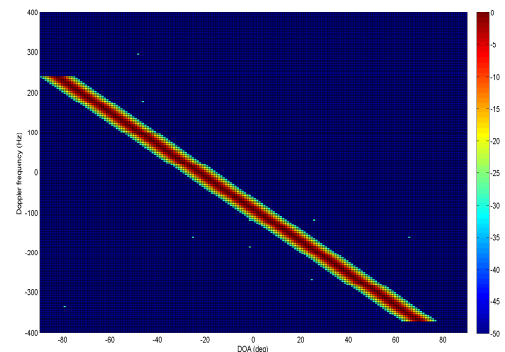

Fig. 2. Estimated sparse radar scene using the D3SR method: ten randomly distributed targets

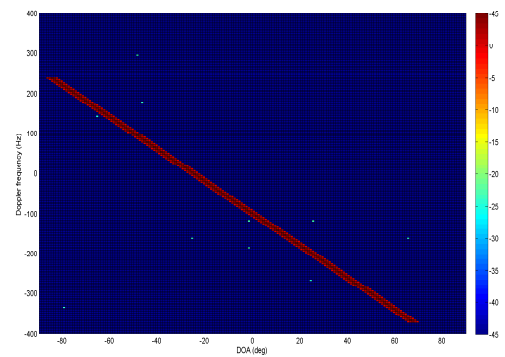

Fig. 3. Estimated sparse radar scene using the proposed method: ten randomly distributed targets

radar scenes reconstructed by the D3SR method and the proposed method, respectively. From Fig. 2, it can be seen that the D3SR method wrongly recovers the elements near the clutter ridge besides the clutter scatters in the DOA-Doppler plane. The targets near the clutter ridge are submerged in the wrongly recovered nearby elements. This is due to the reason that the columns in the original sensing matrix corresponding to the elements near the clutter are highly coherent (similar) with the columns corresponding to the clutter scatters. The D3SR method can not distinguish the highly coherent columns and assigns large values to their corresponding elements. However, the proposed method can estimate the weak elements (targets) as well as the prominent elements (the clutter) accurately, and it can distinguish the targets from clutter successfully in the DOA-Doppler plane (Fig. 3). This verifies that the proposed noise driven compressed sensing method can cope with the deterministic sensing matrix with high coherence efficiently.

Fig. 4 indicates the variation of average reconstruction error $\left(\chi_{\text {average }}=\right.$ $\left.\Sigma_{i=1}^{N_{M C}} \chi^{i} / N_{M C}\right)$ with SNR varying from $0 \mathrm{~dB}$ to $30 \mathrm{~dB}$, which shows that the proposed method is resilient to the measurement noise. The proposed algorithm can do perfect detection $(<0.1)$ of ten targets with measurement noise when the SNR is above $20 \mathrm{~dB}$. For the D3SR method, large reconstruction error $(>2)$ exists even when the SNR exceeds $20 \mathrm{~dB}$.

Moreover, the proposed algorithm is compared with several classic STAP algorithms, e.g. the SMI method, the ADC method and the D3SR method, using the improvement factor loss, which is defined as [14],

$$
I F_{\text {Loss }}=\frac{S C R_{\text {out }} / S C R_{\text {in }}}{\left(S C R_{\text {out }} / S C R_{\text {in }}\right)_{\text {opt }}},
$$




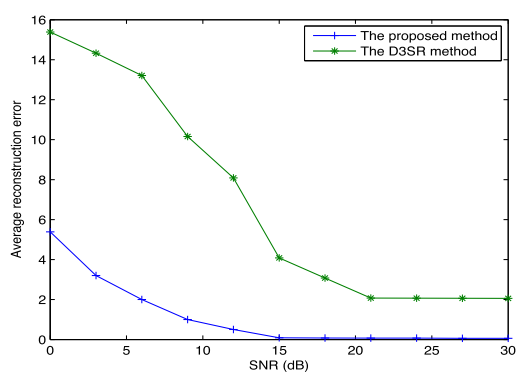

Fig. 4. Reconstruction performance (average reconstruction error) of the proposed method and the D3SR method with varying SNRs

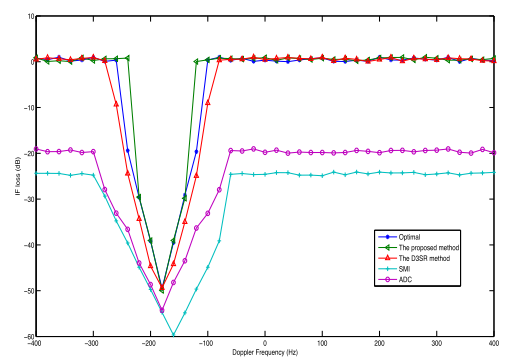

Fig. 5. IF Loss performance of different STAP methods at a DOA angle of $20^{\circ}$ as a function of the Doppler frequency

where $S C R_{\text {out }}$ and $S C R_{\text {in }}$ denote the output signal-clutter-ratio (SCR) and the input SCR respectively. A classic simulation setup in STAP simulations is adopted, where a moving target is coming with a DOA angle of $20^{\circ}$. Different output SCRs are then considered with varying Doppler frequencies. Fig. 5 gives the $I F_{\text {Loss }}$ performance of different STAP algorithms. Because the SCR improvement is mostly achieved in the subspace orthogonal to the clutter, all the STAP methods (the SMI, ADC and D3SR methods) suffer considerable degradation near the clutter notch, no matter what size the total space (i.e., system DOF) is. However, the proposed noise driven compressed sensing algorithm detects the target directly based on the reconstructed radar scene, and it achieves the comparable performance with the optimal filter.

\section{Conclusion}

In this paper, a novel method named noise driven compressed sensing, is proposed to detect unknown moving targets in strong clutter situation directly on the space-time data. The proposed method can estimate the weak elements (targets) as well as the prominent elements (clutter) accurately, and it can distinguish the targets from clutter successfully in the sparse radar scene (the DOA-Doppler plane). 